\title{
Should patients with epistaxis and nasal packing be admitted during the COVID-19 Pandemic? A retrospective study of 885 adult cases with epistaxis.
}

\author{
Vasileios Lachanas ${ }^{1}$, Nick Kalogritsas ${ }^{1}$, Georgios Kontorinis ${ }^{2}$, ANNA MPOURONIKOU ${ }^{1}$, \\ Jiannis Hajiioannou ${ }^{1}$, Fani Saini ${ }^{1}$, Hara Maiou ${ }^{1}$, and Charalampos Skoulakis ${ }^{1}$ \\ ${ }^{1}$ University Hospital of Larissa \\ ${ }^{2}$ Institute of Neurological Sciences, Queen Elizabeth University Hospital
}

January 26, 2021

\begin{abstract}
Key point: * Being one of the commonest presenting emergencies, epistaxis can increase the pressure on the health service; in times similar to COVID-19 pandemic such pressure can be crucial and challenging to deal with. * Non-absorbable nasal packing and home discharge for selected patients can be a safe and efficient alternative treatment method with low readmission rates (5.3\%); such management can be of tremendous help when the hospital capacity is overwhelmed. * Although such protocols can be challenging to generalize, the presented protocol seems safe, easily adoptable without any significant complications.
\end{abstract}

\section{Key points:}

- Being one of the commonest presenting emergencies, epistaxis can increase the pressure on the health service; in times similar to COVID-19 pandemic such pressure can be crucial and challenging to deal with.

- Non-absorbable nasal packing and home discharge for selected patients can be a safe and efficient alternative treatment method with low readmission rates $(5.3 \%)$; such management can be of tremendous help when the hospital capacity is overwhelmed.

- Although such protocols can be challenging to generalize, the presented protocol seems safe, easily adoptable without any significant complications.

\section{Introduction}

Epistaxis occurs in approximately $60 \%$ of people worldwide at least once in their lifetime representing the second commonest cause of Otolaryngology emergency admissions ${ }^{1-5}$. On the other hand, COVID-19 pandemic has spawned an unprecedented crisis overwhelming most healthcare systems globally. Recommendations for epistaxis management during the COVID-19 pandemic suggest the use of absorbable nasal packing or cauterization in order to avoid hospital admissions and visits ${ }^{6}$. However, there are cases where non-absorbable nasal packing is needed and the patient has to be admitted, challenging the hospital capacity, increasing the burden on the health system and potentially exposing the patient to increased risk for COVID-19 infection.

Despite previously used protocol for the management of epistaxis in our department we selected the "Dundee epistaxis protocol" 1 as our new "standard" in 2015. We modified our practice and scheduled an audit, which showed a great improvement in our practice; cautery became the main modality, nasal packings were reduced significantly while all patients with non-absorbable packings were admitted, and "Dundee protocol" was fully adapted in 2016 (unpublished data). During the second wave of COVID-19 pandemic in our area, a high patient influx was noted and the high need for hospitalization of COVID patients resulted in a dramatic 
reduction of available non-COVID hospital beds in all specialties including Otolaryngology. Thus, we rereviewed our audit data in order to see if we can safely reduce epistaxis admissions during the pandemic by discharging patients with packing. Herein we present our experience with dealing with epistaxis with nasal packing on outpatient settings with main focus on the feasibility and safety of such management as well as on how such protocol could relieve the pressure on the inpatient service during the pandemic.

\section{Material and Methods}

We carried out a retrospective case series study on academic, tertiary referral settings. The project was approved by the local University Audit Committee, all participants' data were handled complying with the regulations of University Hospital of Larissa scientific committee and according to the Helsinki and the Health Insurance Portability and Accountability (HIPAA). STROBE guideline has been followed in this study. All cases presenting with epistaxis at the ENT-emergency department within the period January 2011-May 2015 were identified from the audit database and the ENT-emergency register.

We specifically identified the cases that we discharged home (the home group) with nasal packing. We collected demographics, relevant medications, namely coagulation medication, packing material, complications and need for admission.

We included in the 'home group' patients who underwent nasal packing with a non-absorbable material. Patients were only admitted in cases of uncontrolled bleeding (need for surgical intervention), abnormal vital signs, posterior packing, recurrent epistaxis from the "home packing group", haemoglobin $<10 \mathrm{~g} / \mathrm{dl}$, uncontrolled hypertension and high cardiac risk, and in some cases for social reasons (distance from hospital, lack of social support). All 'home patients' were living within a 45 minute driving distance to our centre. Written instructions and contact details were handed out to all patients; these were the safety criteria for discharging the patients. Patients were reviewed 48 hours later on outpatient settings for packing removal.

The nasal examination and the management including the nasal packing was performed by the staff of the department of ENT surgery who were competent dealing with such cases.

\section{Results}

We found a total of 885 adult epistaxis cases in our database (Table 1). We discharged home 772 patients while 113 were admitted. Two hundred and twenty (220) patients were treated with only ice and pressure and discharged; 84 patients treated with cautery ( 80 chemical/4 bipolar) and discharged. Absorbable anterior packing was used in 15 cases; 14 discharged and 1 was admitted. Non-absorbable anterior packing was used in 519 patients $(58.6 \%$ of all cases); 454 of them $(87.5 \%)$ were discharged home with packing (home patient group). Merocel@ (Medtronic XOMED, Jacksonville, FL, USA) was used in 294 and gauge in 160 patients. In all cases packing was soaked in mupirocin nasal ointment in order to avoid toxic shock syndrome and in all cases was removed within 48 hours in ENT emergency department. All "home patients" fulfilled the inclusion criteria as above. Posterior packing was used in 47 patients and all of them were admitted.

From the "home patients" with non-absorbable packing 12 were receiving antiplatelet and 10 anticoagulant therapy. Antiplatelet therapy was not withdrawn while in all patients under anticoagulant therapy INR was within therapeutic range.

Bleeding recurrence occurred in 24 patients (5.3\%) of the home group. These patients were treated as inpatients; 3 with cautery, 7 with non-absorbable anterior packing, 9 with posterior packing, and 5 with surgical intervention as necessary. Interesting, no recurrence occurred in the antiplatelet/anticoagulant subgroup.

No packing related complications were noticed in "home patients".

During the 'second wave' of COVID-19-pandemic we have followed this policy in 24 patients who consented to be discharged home. It should be noticed that, above others all of them were afraid the risk of COVID-19 contagion by staying in the hospital; none of the 24 patients had to be admitted.

\section{Discussion}


We present a safe and efficient approach to managing selected patients with epistaxis on an outpatient basis, showing only $5 \%$ re-admission rate. This approach can be of importance during the COVID-19 pandemic as it can significantly help with hospital bed capacity; in a similar way, it can facilitate health system pressure during busy periods.

We have found very few studies regarding discharging patients with epistaxis and non-absorbable packing.

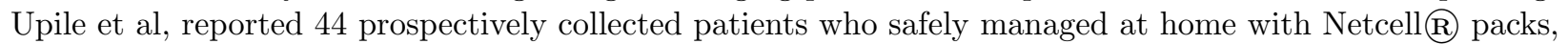
reducing the number of admissions by $73 \%$. Epistaxis recurrence was their main unwanted event (seven cases) while antiplatelet/anticoagulation therapy did not appear to be a barrier to discharge ${ }^{3,4}$. Van Wyk et al, in a retrospective study of 116 patients with epistaxis reported 46 patients discharged with nasal packing in situ, only $16 \%$ returned due to re-bleeding, while no other adverse effects were recorded ${ }^{5}$. Our data, arising from a more extensive case series, demonstrate that selected cases with non-absorbable anterior nasal packing may be safely discharged home and a high decrease in hospital admissions can be achieved, while re-bleeding occurred in only $5.3 \%$ of our cases. While one could argue that these patients need to represent for packing removal, the health system resources needed for an outpatient appointment are not comparable to an inpatient admission.

Interestingly, based on our data, patients receiving antiplatelet therapy or anticoagulation within the therapeutic range can also be treated home; however, there was only a small number of such patients, which can explain our results. On these grounds safe, generalized conclusions cannot be made for this patient subgroup; careful clinical assessment and decision making is important for this subgroup.

Despite the relatively large number of cases, the retrospective nature of our study is the main limitation of our study. We used a robust database of epistaxis admissions/ presentation in a unicentral ENT emergency department and relevant details to minimize such bias and present meaningful results.

In conclusion, based on our results, non-absorbable packing and planned representation for packing removal on outpatient settings is a safe way of managing selected patients with epistaxis; in circumstances like COVID19-pandemic such management can reduce hospital admissions and relieve the pressure on the health system without compromising the outcome.

Conflict of interest: None to declare.

Data availability statement: The data that support the findings of this study are available on request from the corresponding author. The data are not publicly available due to privacy or ethical restrictions.

\section{References}

1. Barnes ML, Spielmann PM, White PS. Epistaxis: a contemporary evidence based approach. Otolaryngol Clin North Am. 2012;45(5):1005-17. doi: 10.1016/j.otc.2012.06.018.

2. Spielmann PM, Barnes ML, White PS. Controversies in the specialist management of adult epistaxis: an evidence-based review. Clin Otolaryngol. 2012;37(5):382-9. doi: 10.1111/coa.12024.

3. Upile T, Jerjes W, Sipaul F, El Maaytah M, Nouraei SA, Singh S, Hopper C, Wright A. The role of surgical audit in improving patient management; nasal haemorrhage: an audit study. BMC Surg. 2007; 7:19. doi: 10.1186/1471-2482-7-19.

4. Upile T, Jerjes W, Sipaul F, Maaytah ME, Singh S, Hopper C, Wright A. A change in UK epistaxis management. Eur Arch Otorhinolaryngol. 2008;265(11):1349-54. doi: 10.1007/s00405-008-0657-1.

5. Van Wyk FC, Massey S, Worley G, Brady S. Do all epistaxis patients with a nasal pack need admission? A retrospective study of 116 patients managed in accident and emergency according to a peer reviewed protocol. J Laryngol Otol. 2007;121(3):222-7. doi: 10.1017/S0022215106003148.

6. D'Aguanno V, Ralli M, Greco A, de Vincentiis M. Clinical Recommendations for Epistaxis Management During the COVID-19 Pandemic. Otolaryngol Head Neck Surg. 2020;163(1):75-77. doi: $10.1177 / 0194599820926497$.

\section{Hosted file}


table 1.pdf available at https://authorea.com/users/391825/articles/505827-shouldpatients-with-epistaxis-and-nasal-packing-be-admitted-during-the-covid-19-pandemic-aretrospective-study-of-885-adult-cases-with-epistaxis 